\title{
Hamilton-Poisson Realizations of the Integrable Deformations of the Rikitake System
}

\author{
Cristian Lăzureanu \\ Department of Mathematics, Politehnica University of Timişoara, Piaţa Victoriei No. 2, 300006 Timişoara, Romania \\ Correspondence should be addressed to Cristian Lăzureanu; cristian.lazureanu@upt.ro
}

Received 30 April 2017; Revised 19 June 2017; Accepted 5 July 2017; Published 27 August 2017

Academic Editor: Ricardo Weder

Copyright (C) 2017 Cristian Lăzureanu. This is an open access article distributed under the Creative Commons Attribution License, which permits unrestricted use, distribution, and reproduction in any medium, provided the original work is properly cited.

\begin{abstract}
Integrable deformations of an integrable case of the Rikitake system are constructed by modifying its constants of motions. Hamilton-Poisson realizations of these integrable deformations are given. Considering two concrete deformation functions, a Hamilton-Poisson approach of the obtained system is presented. More precisely, the stability of the equilibrium points and the existence of the periodic orbits are proved. Furthermore, the image of the energy-Casimir mapping is determined and its connections with the dynamical elements of the considered system are pointed out.
\end{abstract}

\section{Introduction}

In 1958, Rikitake examined the behavior of two disk dynamos coupled one to another in relation to Earth's magnetic field [1]. We mention that there are some other nonlinear disk dynamo systems (see, e.g., [2-4]). The Rikitake system has chaotic behavior [5], but one may consider integrable cases of this system [6-8]. The Rikitake system was widely analyzed. We recall some papers on the dynamics, control, synchronization, and secure communication of Rikitake chaotic system, namely, [9-14]. Moreover, hyperchaotic Rikitake systems were considered (see, e.g., $[15,16]$ ).

The study of an integrable version of the Rikitake system from some standard and nonstandard Poisson geometry points of view was given in [17]. Because this system is considered in our paper, we mention some of its dynamical properties: there are three families of equilibrium points and also periodic orbits around the stable equilibrium points and homoclinic and heteroclinic orbits. The same properties and the new ones are obtained considering some parametric controls $[18,19]$.

Recently, constructions of integrable deformations of a given integrable system were given. In [20], modifying the constants of motion, integrable deformations of the Euler top were obtained. Using the same technique, in [21], integrable deformations of the three-dimensional Maxwell-Bloch equations were analyzed. In [22], integrable deformations of a class of three-dimensional Lotka-Volterra equations induced from the coproduct map were presented. In [23], considering Poisson-Lie groups as deformations of Lie-Poisson (co)algebras, integrable deformations of some integrable types of Rössler and Lorenz systems were considered.

Using the method given in [20], in this paper, we construct integrable deformations of the integrable version of the Rikitake system considered in [17]. We prove that these integrable deformations have Hamilton-Poisson realizations, which allows us to study them from some standard and nonstandard Poisson geometry points of view. The study of a dynamical system from standard Poisson geometry point of view means the study of its dynamical elements, such as equilibrium points, periodic orbits, and homoclinic and heteroclinic orbits, as well as the dynamical behavior, that is, stability, bifurcation phenomena, periodic motion, and homoclinic and heteroclinic connections. The study of a dynamical system that has a Hamilton-Poisson realization from nonstandard Poisson geometry point of view means the study of the connections between the above-mentioned dynamical properties and the geometry of the image of the energy-Casimir mapping associated with the considered system [17].

The paper is organized as follows. In Section 2, we construct integrable deformations of an integrable version of the 
Rikitake system by modifying its constants of motion. In Section 3, we obtain two Hamilton-Poisson realizations of the new system. Moreover, we prove that the obtained system has infinitely many Hamilton-Poisson realizations. In Section 4, we consider a particular integrable deformation of the Rikitake system. We remark that this system is in fact a controlled system obtained by applying two parametric controls to the Rikitake system such that all the equilibrium points are nonlinearly stable and all the trajectories are periodic orbits (for details about parametric controls see, e.g., [24]). In other words, the controlled Rikitake system may be nonlinearly stabilized about its equilibrium points. We also present the connections between the energy-Casimir mapping and the above-mentioned dynamical elements. Concluding remarks are made in Section 5.

\section{Integrable Deformations of the Rikitake System}

Using the method considered in [20], in this section, we construct integrable deformations of the Rikitake system.

We recall that "the Rikitake two-disk dynamo model [1] consists of two connected identical frictionless disk dynamos. The dynamos are driven by identical torques $G$ to maintain their motions in the face of Ohmic losses in the coils and disks. The equations describing the system are given by the nonlinear dynamical system

$$
\begin{aligned}
\frac{d I_{1}}{d t} & =\frac{M}{L} \Omega_{1}(t) I_{2}(t)-\frac{R}{L} I_{1}(t), \\
\frac{d I_{2}}{d t} & =\frac{M}{L} \Omega_{2}(t) I_{1}(t)-\frac{R}{L} I_{2}(t), \\
\frac{d \Omega_{1}}{d t} & =-\frac{M}{C} I_{1}(t) I_{2}(t)+\frac{G}{C}, \\
\frac{d \Omega_{2}}{d t} & =-\frac{M}{C} I_{1}(t) I_{2}(t)+\frac{G}{C},
\end{aligned}
$$

where $I_{1}$ and $I_{2}$ are the currents, $\Omega_{1}$ and $\Omega_{2}$ are the angular velocities, $L$ is the self-inductance, $R$ is the resistance associated with each dynamo and its connecting circuitry, $M$ is the mutual inductance between the dynamo circuits, and $C$ is the moment of inertia of a dynamo about its axis" [25, 26].

Using the transformation

$$
\begin{aligned}
t & =\frac{L}{M} t^{\prime}, \\
I_{1}(t) & =\sqrt{\frac{C}{L}} x\left(t^{\prime}\right), \\
I_{2}(t) & =\sqrt{\frac{C}{L}} y\left(t^{\prime}\right), \\
\Omega_{1}(t) & =z\left(t^{\prime}\right)+a, \\
\Omega_{2}(t) & =z\left(t^{\prime}\right)-a,
\end{aligned}
$$

where $a$ is a real parameter, system (1) becomes

$$
\begin{aligned}
& \frac{d x}{d t^{\prime}}=y z+a y-\frac{R}{M} x, \\
& \frac{d y}{d t^{\prime}}=x z-a x-\frac{R}{M} y, \\
& \frac{d z}{d t^{\prime}}=-x y+\frac{G L}{M C} .
\end{aligned}
$$

Imposing the condition that the above system be conservative, we obtain $R=0$. Therefore we consider in our work the following integrable version of the Rikitake system $(G=0)$ :

$$
\begin{aligned}
& \dot{x}=y z+a y, \\
& \dot{y}=x z-a x, \\
& \dot{z}=-x y
\end{aligned}
$$

where $a \in \mathbb{R}$. Two constants of motion of system (4) are given by

$$
\begin{aligned}
& J_{1}(x, y, z)=\frac{1}{2} x^{2}+\frac{1}{2} y^{2}+z^{2} \\
& J_{2}(x, y, z)=\frac{1}{2} x^{2}-\frac{1}{2} y^{2}+2 a z .
\end{aligned}
$$

In the following, we prove that (4) are uniquely determined by these constants of motion, up to a parametrization of time. Indeed, differentiating the above constants of motion (5), we obtain

$$
\begin{aligned}
& x \dot{x}+y \dot{y}=-2 z \dot{z}, \\
& x \dot{x}-y \dot{y}=-2 a \dot{z} ;
\end{aligned}
$$

hence,

$$
\begin{aligned}
& \dot{x}=-\frac{z+a}{x} \dot{z}, \\
& \dot{y}=-\frac{z-a}{y} \dot{z} .
\end{aligned}
$$

Consider $\dot{z}=-x y f$, where $f=f(t)$ is an arbitrary continuous function. We deduce

$$
\begin{aligned}
& \dot{x}=(y z+a y) f, \\
& \dot{y}=(x z-a x) f, \\
& \dot{z}=-x y f .
\end{aligned}
$$

Using the transformation $t=t(\tau)$, where $\tau$ is the new time variable, given by

$$
\tau=\int_{0}^{t} f(s) d s
$$

we obtain

$$
\begin{aligned}
\frac{d x}{d \tau} & =\frac{d x}{d t} \cdot \frac{d t}{d \tau}=(y z+a y) f \cdot \frac{1}{f} \\
& =y(\tau) z(\tau)+a y(\tau) .
\end{aligned}
$$


Analogously, we obtain

$$
\begin{aligned}
& \frac{d y}{d \tau}=x(\tau) z(\tau)-a x(\tau), \\
& \frac{d z}{d \tau}=-x(\tau) y(\tau)
\end{aligned}
$$

as required.

This property of (4) allows obtaining integrable deformations of the considered Rikitake system altering its constants of motion. More precisely, we consider the new constants of motion $C_{1}$ and $C_{2}$ given by

$$
\begin{aligned}
& C_{1}(x, y, z)=\frac{1}{2} x^{2}+\frac{1}{2} y^{2}+z^{2}+\alpha(x, y, z), \\
& C_{2}(x, y, z)=\frac{1}{2} x^{2}-\frac{1}{2} y^{2}+2 a z+\beta(x, y, z),
\end{aligned}
$$

where $\alpha$ and $\beta$ are arbitrary differentiable functions. By (12)(13), we get

$$
\begin{aligned}
& x \dot{x}+y \dot{y}+2 z \dot{z}+\frac{\partial \alpha}{\partial x} \dot{x}+\frac{\partial \alpha}{\partial y} \dot{y}+\frac{\partial \alpha}{\partial z} \dot{z}=0 \\
& x \dot{x}-y \dot{y}+2 a \dot{z}+\frac{\partial \beta}{\partial x} \dot{x}+\frac{\partial \beta}{\partial y} \dot{y}+\frac{\partial \beta}{\partial z} \dot{z}=0
\end{aligned}
$$

or, equivalently,

$$
\begin{aligned}
& \left(x+\frac{\partial \alpha}{\partial x}\right) \dot{x}+\left(y+\frac{\partial \alpha}{\partial y}\right) \dot{y}=-\left(2 z+\frac{\partial \alpha}{\partial z}\right) \dot{z} \\
& \left(x+\frac{\partial \beta}{\partial x}\right) \dot{x}-\left(y-\frac{\partial \beta}{\partial y}\right) \dot{y}=-\left(2 a+\frac{\partial \beta}{\partial z}\right) \dot{z}
\end{aligned}
$$

Solving this algebraic system, we obtain

$$
\begin{aligned}
\dot{x} & =\frac{y z+a y+a(\partial \alpha / \partial y)+(y / 2)(\partial \alpha / \partial z)-z(\partial \beta / \partial y)+(y / 2)(\partial \beta / \partial z)+(1 / 2)(\partial \alpha / \partial y \cdot \partial \beta / \partial z-\partial \alpha / \partial z \cdot \partial \beta / \partial y)}{-x y-(y / 2)(\partial \alpha / \partial x)-(x / 2)(\partial \alpha / \partial y)-(y / 2)(\partial \beta / \partial x)+(x / 2)(\partial \beta / \partial y)+(1 / 2)(\partial \alpha / \partial x \cdot \partial \beta / \partial y-\partial \alpha / \partial y \cdot \partial \beta / \partial x)} \\
& \cdot \dot{z}, \\
\dot{y}= & \frac{x z-a x-a(\partial \alpha / \partial x)+(x / 2)(\partial \alpha / \partial z)+z(\partial \beta / \partial x)-(x / 2)(\partial \beta / \partial z)-(1 / 2)(\partial \alpha / \partial x \cdot \partial \beta / \partial z-\partial \alpha / \partial z \cdot \partial \beta / \partial x)}{-x y-(y / 2)(\partial \alpha / \partial x)-(x / 2)(\partial \alpha / \partial y)-(y / 2)(\partial \beta / \partial x)+(x / 2)(\partial \beta / \partial y)+(1 / 2)(\partial \alpha / \partial x \cdot \partial \beta / \partial y-\partial \alpha / \partial y \cdot \partial \beta / \partial x)}
\end{aligned}
$$

$$
\dot{z}
$$

We denote by $\dot{z}$ the denominator of the above expressions. Thus, we have constructed the following integrable deformation of the Rikitake system:

$$
\begin{aligned}
\dot{x}= & y z+a y+a \frac{\partial \alpha}{\partial y}+\frac{y}{2} \frac{\partial \alpha}{\partial z}-z \frac{\partial \beta}{\partial y}+\frac{y}{2} \frac{\partial \beta}{\partial z} \\
& +\frac{1}{2}\left(\frac{\partial \alpha}{\partial y} \cdot \frac{\partial \beta}{\partial z}-\frac{\partial \alpha}{\partial z} \cdot \frac{\partial \beta}{\partial y}\right), \\
\dot{y}= & x z-a x-a \frac{\partial \alpha}{\partial x}+\frac{x}{2} \frac{\partial \alpha}{\partial z}+z \frac{\partial \beta}{\partial x}-\frac{x}{2} \frac{\partial \beta}{\partial z} \\
& -\frac{1}{2}\left(\frac{\partial \alpha}{\partial x} \cdot \frac{\partial \beta}{\partial z}-\frac{\partial \alpha}{\partial z} \cdot \frac{\partial \beta}{\partial x}\right), \\
\dot{z}= & -x y-\frac{y}{2} \frac{\partial \alpha}{\partial x}-\frac{x}{2} \frac{\partial \alpha}{\partial y}-\frac{y}{2} \frac{\partial \beta}{\partial x}+\frac{x}{2} \frac{\partial \beta}{\partial y} \\
& +\frac{1}{2}\left(\frac{\partial \alpha}{\partial x} \cdot \frac{\partial \beta}{\partial y}-\frac{\partial \alpha}{\partial y} \cdot \frac{\partial \beta}{\partial x}\right) .
\end{aligned}
$$

It is obvious that if $\alpha$ and $\beta$ are constant functions, then (17) reduces to (4).

\section{Hamilton-Poisson Realizations of the Integrable Deformations of the Rikitake System}

In this section, we give two Hamilton-Poisson realizations of system (17). Furthermore, we obtain that the considered system is bi-Hamiltonian. We also prove that system (17) has infinitely many Hamilton-Poisson realizations.

In order to obtain a Hamilton-Poisson realization

$$
\left(\mathbb{R}^{3},\{\cdot, \cdot\}_{1}, H_{1}\right)
$$

of system (17), we construct a Poisson bracket

$$
\{\cdot, \cdot\}_{1}: C^{\infty}\left(\mathbb{R}^{3}, \mathbb{R}\right) \times C^{\infty}\left(\mathbb{R}^{3}, \mathbb{R}\right) \longrightarrow C^{\infty}\left(\mathbb{R}^{3}, \mathbb{R}\right),
$$

considering that $C_{1}$ (see (12)) is a Casimir function; that is, $\left\{f, C_{1}\right\}_{1}=0$, for any function $f \in C^{\infty}\left(\mathbb{R}^{3}, \mathbb{R}\right)$, or $\Pi_{1} \cdot \nabla C_{1}=\mathbf{0}$, where $\Pi_{1}$ is the matrix of $\{\cdot, \cdot\}_{1}$; namely,

$$
\Pi_{1}=\left[\begin{array}{ccc}
0 & \{x, y\}_{1} & \{x, z\}_{1} \\
-\{x, y\}_{1} & 0 & \{y, z\}_{1} \\
-\{x, z\}_{1} & -\{y, z\}_{1} & 0
\end{array}\right] .
$$


We obtain

$$
\begin{aligned}
& \left(y+\frac{\partial \alpha}{\partial y}\right)\{x, y\}_{1}+\left(2 z+\frac{\partial \alpha}{\partial z}\right)\{x, z\}_{1}=0, \\
& \left(x+\frac{\partial \alpha}{\partial x}\right)\{x, y\}_{1}=\left(2 z+\frac{\partial \alpha}{\partial z}\right)\{y, z\}_{1}, \\
& \left(x+\frac{\partial \alpha}{\partial x}\right)\{x, z\}_{1}=-\left(y+\frac{\partial \alpha}{\partial y}\right)\{y, z\}_{1} .
\end{aligned}
$$

We also impose the condition that $H_{1}:=C_{2}$ (13) be the Hamiltonian of system (17); namely, $\Pi_{1} \cdot \nabla C_{2}=(\dot{x}, \dot{y}, \dot{z})^{t}$ :

$$
\begin{aligned}
& \left(-y+\frac{\partial \beta}{\partial y}\right)\{x, y\}_{1}+\left(2 a+\frac{\partial \beta}{\partial z}\right)\{x, z\}_{1}=\dot{x} \\
& -\left(x+\frac{\partial \beta}{\partial x}\right)\{x, y\}_{1}+\left(2 a+\frac{\partial \beta}{\partial z}\right)\{y, z\}_{1}=\dot{y} \\
& -\left(x+\frac{\partial \beta}{\partial x}\right)\{x, z\}_{1}-\left(-y+\frac{\partial \beta}{\partial y}\right)\{y, z\}_{1}=\dot{z} .
\end{aligned}
$$

Considering (21)-(22) as an algebraic system and using (17), we obtain

$$
\begin{aligned}
& \{x, y\}_{1}=-z-\frac{1}{2} \cdot \frac{\partial \alpha}{\partial z} \\
& \{x, z\}_{1}=\frac{y}{2}+\frac{1}{2} \cdot \frac{\partial \alpha}{\partial y} \\
& \{y, z\}_{1}=-\frac{x}{2}-\frac{1}{2} \cdot \frac{\partial \alpha}{\partial x} .
\end{aligned}
$$

The bracket $\{\cdot, \cdot\}_{1}$ verifies the Jacobi identity; thus it is a Poisson bracket. We have proven the following result.

Proposition 1. System (17) has the Hamilton-Poisson realization

$$
\left(\mathbb{R}^{3},\{\cdot, \cdot\}_{1}, H_{1}\right)
$$

where the Poisson bracket $\{\cdot, \cdot\}_{1}$ is given by (23) and $H_{1}:=C_{2}$ given by (13) is the Hamiltonian.

Similarly, we obtain the second Poisson structure $\{\cdot, \cdot\}_{2}$. More precisely, imposing the conditions that $C_{2}$ be a Casimir function and $C_{1}$ the Hamiltonian, we get

$$
\begin{aligned}
& \{x, y\}_{2}=a+\frac{1}{2} \cdot \frac{\partial \beta}{\partial z} \\
& \{x, z\}_{2}=\frac{y}{2}-\frac{1}{2} \cdot \frac{\partial \beta}{\partial y} \\
& \{y, z\}_{2}=\frac{x}{2}+\frac{1}{2} \cdot \frac{\partial \beta}{\partial x}
\end{aligned}
$$

Consequently, we can state the next result.
Proposition 2. System (17) has the Hamilton-Poisson realization

$$
\left(\mathbb{R}^{3},\{\cdot, \cdot\}_{2}, H_{2}\right)
$$

where the Poisson bracket $\{\cdot, \cdot\}_{2}$ is given by (25) and $H_{2}:=C_{1}$ given by (12) is the Hamiltonian.

Remark 3. System (17) has the form $\Pi_{1} \cdot \nabla C_{2}=\Pi_{2} \cdot \nabla C_{1}=$ $(\dot{x}, \dot{y}, \dot{z})^{t}$. Because $\Pi_{1}+\Pi_{2}$ is a Poisson structure, the Poisson brackets $\{\cdot, \cdot\}_{1}$ and $\{\cdot, \cdot\}_{2}$ are compatible. Consequently, system (17) is a bi-Hamiltonian system.

The last result from this section furnishes other Hamilton-Poisson realizations of system (17).

Proposition 4. System (17) has infinitely many HamiltonPoisson realizations given by

$$
\left(\mathbb{R}^{3},\{\cdot, \cdot\}_{a, b}, H_{c, d}\right), \quad a, b, c, d \in S L(2, \mathbb{R}),
$$

where

$$
\begin{gathered}
\{\cdot, \cdot\}_{a, b}=a\{\cdot, \cdot\}_{1}+b\{\cdot, \cdot\}_{2}, \\
H_{c, d}=-c C_{1}+d C_{2} .
\end{gathered}
$$

Proof. Taking into account the fact that $\Pi_{1}+\Pi_{2}$ is a Poisson structure, we deduce that $\Pi_{a, b}=a \Pi_{1}+b \Pi_{2}$ is a Poisson structure for every $a, b \in \mathbb{R}$. Considering $c, d \in \mathbb{R}$ such that $a d-b c=1$ and the functions $H_{c, d}=-c C_{1}+d C_{2}$ and $C_{a, b}=$ $a C_{1}-b C_{2}$, we have $\Pi_{a, b} \cdot \nabla C_{a, b}=\mathbf{0}$, and $\Pi_{a, b} \cdot \nabla H_{c, d}=(\dot{x}, \dot{y}, \dot{z})^{t}$, which is what we set out to prove.

We remark that, in the geometric frame given by the above Hamilton-Poisson realizations, a Hamilton-Poisson approach of system (17) may be done. We exemplify in Section 4.

\section{A Particular Integrable Deformation of the Rikitake System}

In this section, we consider particular functions $\alpha$ and $\beta$, and we analyze the dynamics of system (17) in this particular case. We study the stability of the equilibrium points, and we prove the existence of the periodic orbits around the nonlinearly stable equilibrium points. We also give some properties of the energy-Casimir mapping associated with the considered system.

We consider the functions $\alpha(x, y, z)=0$ and $\beta(x, y, z)=$ $k / x^{2}$, where $k \in(-\infty, 0)$ is a deformation parameter. Then system (17) becomes

$$
\begin{aligned}
& \dot{x}=y z+a y, \\
& \dot{y}=x z-a x-\frac{2 k z}{x^{3}}, \\
& \dot{z}=-x y+\frac{k y}{x^{3}} .
\end{aligned}
$$


We observe that system (29) is invariant under the transformation $(x, y, z) \rightarrow(-x,-y, z)$. Therefore, we analyze this system in the case where $x \in(0, \infty)$.

We notice that system (29) may be regarded as the Rikitake system (4) with two parametric controls $u_{1}(x$, $y, z)=-2 k z / x^{3}$ and $u_{2}(x, y, z)=k y / x^{3}$ about $O y$ and $\mathrm{O} z$ axes, respectively, where $k \in(-\infty, 0)$ is the tuning parameter.

The aim of this section is to study the above particular integrable deformation of the Rikitake system from the Poisson geometry point of view. The first step in this approach is to give a Hamilton-Poisson realization of this system, as in Section 3. The constants of motion of system (29) are given by

$$
\begin{aligned}
& C(x, y, z)=\frac{1}{2} x^{2}+\frac{1}{2} y^{2}+z^{2}, \\
& H(x, y, z)=\frac{1}{2} x^{2}+\frac{k}{x^{2}}-\frac{1}{2} y^{2}+2 a z,
\end{aligned}
$$

and considering $C$ a Casimir function and $H$ the Hamiltonian, the corresponding Poisson bracket is given by (23):

$$
\begin{aligned}
& \{x, y\}_{1}=-z, \\
& \{x, z\}_{1}=\frac{y}{2}, \\
& \{y, z\}_{1}=-\frac{x}{2} .
\end{aligned}
$$

We remark that this Poisson bracket is linear; therefore, it is in fact a Lie-Poisson bracket on the dual of certain Lie algebra, namely, $\mathfrak{S} \mathfrak{o}(3)^{*}$. Indeed, consider Lie group

$$
\begin{aligned}
& S O(3) \\
& \quad=\left\{A \in G L(3, \mathbb{R}) \mid A A^{t}=A^{t} A=I_{3}, \operatorname{det} A=1\right\}
\end{aligned}
$$

and its corresponding Lie algebra

$$
\begin{aligned}
\mathfrak{g} \mathfrak{o}(3) & =\{X \in g l(3, \mathbb{R}) \mid X \\
& \left.=\left[\begin{array}{ccc}
0 & -w & v \\
w & 0 & -u \\
-v & u & 0
\end{array}\right], u, v, w \in \mathbb{R}\right\},
\end{aligned}
$$

with the commutator bracket $[X, Y]=X Y-Y X$. As a real vector space, the Lie algebra $\mathfrak{G o}(3)$ is generated by the basis $B_{\mathfrak{g} \mathfrak{o}(3)}=\left\{f_{1}, f_{2}, f_{3}\right\}$, where

$$
f_{1}=\left[\begin{array}{ccc}
0 & 0 & 0 \\
0 & 0 & -1 \\
0 & 1 & 0
\end{array}\right]
$$

$$
\begin{aligned}
& f_{2}=\left[\begin{array}{ccc}
0 & 0 & 1 \\
0 & 0 & 0 \\
-1 & 0 & 0
\end{array}\right], \\
& f_{3}=\left[\begin{array}{lll}
0 & -1 & 0 \\
1 & 0 & 0 \\
0 & 0 & 0
\end{array}\right] .
\end{aligned}
$$

Following [27], we consider the nonstandard commutator $[\cdot, \cdot]_{D}$ on the space of skew-symmetric matrices $\mathfrak{g} \mathfrak{o}(3)$ given by

$$
[X, Y]_{D}=X D Y-Y D X,
$$

where $D=\operatorname{diag}(-1 / 2,-1 / 2,-1)$ is a diagonal matrix. We obtain

$$
\begin{aligned}
& {\left[f_{1}, f_{2}\right]_{D}=-f_{3},} \\
& {\left[f_{1}, f_{3}\right]_{D}=\frac{1}{2} f_{2},} \\
& {\left[f_{2}, f_{3}\right]_{D}=-\frac{1}{2} f_{1} .}
\end{aligned}
$$

Thus, on $\mathfrak{g} \mathfrak{o}(3)^{*} \simeq \mathbb{R}^{3}$, the Lie-Poisson structure $\{\cdot, \cdot\}_{1}$ (see (31)) is defined. As a consequence, system (29) has the Hamilton-Poisson realization $\left(\mathfrak{g o}(3)^{*},\{\cdot, \cdot\}_{1}, H\right)$.

The next step in our approach is to study the stability of the equilibrium points of the considered system. We establish the nonlinear stability using Lyapunov function [28].

The equilibrium points of system (29) are given by the family

$$
\mathscr{E}=\left\{\left(M, 0, \frac{a M^{4}}{M^{4}-2 k}\right): M \in(0, \infty)\right\},
$$

and their stability is presented in the next result.

Proposition 5. All the equilibrium points of the family $\mathscr{E}$ are nonlinearly stable.

Proof. Let $e_{M}:=\left(M, 0, a M^{4} /\left(M^{4}-2 k\right)\right)$ be an equilibrium point of system (29), where $M>0$, and let $V$ be a neighborhood of $e_{M}$ such that $x>0$ for every $(x, y, z) \in V$. Then the function $L: V \rightarrow \mathbb{R}$, where

$$
\begin{aligned}
L(x, y, z)= & \frac{-k}{M^{4}-2 k}\left(x-\frac{M^{2}}{x}\right)^{2}+\frac{M^{4}-k}{M^{4}-2 k} y^{2} \\
& +\left(z-\frac{a M^{4}}{M^{4}-2 k}\right)^{2},
\end{aligned}
$$

has the following properties:

(i) $L(x, y, z) \geq 0$, for every $(x, y, z) \in V$.

(ii) $L(x, y, z)=0$ if and only if $(x, y, z)=e_{M}$.

(iii) The derivative of $L$ along the solution $(x(t), y(t), z(t))$ of system (29) vanishes. 
Indeed, assertions (i) and (ii) follow by the condition that $k<0$. For (iii), we have

$$
\begin{aligned}
\dot{L}(x, y, z) & =\frac{\partial L}{\partial x} \cdot \dot{x}+\frac{\partial L}{\partial y} \cdot \dot{y}+\frac{\partial L}{\partial z} \cdot \dot{z} \\
= & \frac{-2 k}{M^{4}-2 k}\left(x-\frac{M^{2}}{x}\right)\left(1+\frac{M^{2}}{x^{2}}\right)(y z+a y) \\
& +2 \frac{M^{4}-k}{M^{4}-2 k} y\left(x z-a x-\frac{2 k z}{x^{3}}\right) \\
& +2\left(z-\frac{a M^{4}}{M^{4}-2 k}\right)\left(-x y+\frac{k y}{x^{3}}\right)=0
\end{aligned}
$$

for all $(x, y, z) \in V$, where we have used (29). Therefore, by [28], we deduce that the equilibrium point $e_{M}$ is nonlinearly stable. Thus, all the equilibrium points of the family $\mathscr{E}$ are nonlinearly stable.

Now, we study the existence of the periodic orbits of system (29) around nonlinearly stable equilibrium points. We apply a version of Weinstein-Moser result in the case of zero eigenvalue, namely, Theorem 2.1 from [29], which ensures the existence of periodic orbits around an equilibrium point. We recall this result.

Theorem. Let $\dot{x}=X(x)$ be a dynamical system on a differentiable manifold $U$, let $x_{0}$ be an equilibrium point, that is, $X\left(x_{0}\right)=0$, and let $C:=\left(C_{1}, \ldots, C_{j}\right): U \rightarrow \mathbb{R}^{j}$ be a vector valued constant of motion for the above dynamical system with $C\left(x_{0}\right)$ being a regular value for $C$. If

(i) the eigenspace corresponding to the eigenvalue zero of the linearized system around $x_{0}$ has dimension $j$,

(ii) $D X\left(x_{0}\right)$ has a pair of pure complex eigenvalues $\pm i \omega$ with $\omega \neq 0$,

(iii) there exist a constant of motion $I: U \rightarrow \mathbb{R}$ for the vector field $X$ with $d I\left(x_{0}\right)=0$ such that $\left.d^{2} I\left(x_{0}\right)\right|_{W \times W}>0$, where $W=\bigcap_{l=1}^{j} \operatorname{ker} d C_{l}\left(x_{0}\right)$,

then for each sufficiently small $\varepsilon>0$ any integral surface $I(x)=I\left(x_{0}\right)+\varepsilon^{2}$ contains at least one periodic solution of $X$ whose period is close to the period of the corresponding linear system around $x_{0}$.

In our case, we have the following.

Proposition 6. Let $e_{M}:=\left(M, 0, a M^{4} /\left(M^{4}-2 k\right)\right) \in \mathscr{E}, a \in$ $\mathbb{R}, k \in(-\infty, 0)$. Then, for each sufficiently small $\varepsilon \in(0, \infty)$, any integral surface

$$
\begin{aligned}
\Sigma_{\varepsilon}^{M}: & \frac{-k}{M^{4}-2 k}\left(x-\frac{M^{2}}{x}\right)^{2}+\frac{M^{4}-k}{M^{4}-2 k} y^{2} \\
& +\left(z-\frac{a M^{4}}{M^{4}-2 k}\right)^{2}=\varepsilon^{2}
\end{aligned}
$$

contains around $e_{M}$ at least one periodic orbit $\gamma_{\varepsilon}^{e_{M}}$ of system (29) whose period is close to $2 \pi / \omega$, where $\omega=$ $\sqrt{\left(M^{4}-k\right)\left[\left(M^{4}-2 k\right)^{3}-16 k a^{2} M^{6}\right]} / M^{3}\left(M^{4}-2 k\right)$.

Proof. We consider $j=1$ and $C:(0, \infty) \times \mathbb{R} \times \mathbb{R} \rightarrow \mathbb{R}$ given by (30) in the above-mentioned theorem. The linearization of system (29) at $e_{M}$ has the eigenvalues $\lambda_{1}=0$ and $\lambda_{2,3}=$ $\pm i \omega$, and the eigenspace corresponding to the eigenvalue $\lambda_{1}$ is $\operatorname{span}_{\mathbb{R}}\left\{\left(\left(M^{4}-2 k\right)^{2}, 0,-8 a k M^{3}\right)\right\}$. Furthermore,

$$
d C\left(e_{M}\right)=M d x+\frac{2 a M^{4}}{M^{4}-2 k} d z
$$

hence,

$$
\begin{aligned}
W & =\operatorname{ker} d C\left(e_{M}\right) \\
& =\operatorname{span}_{\mathbb{R}}\left\{(0,1,0),\left(-2 a M^{3}, 0, M^{4}-2 k\right)\right\} .
\end{aligned}
$$

The function $L$ given by (38) is a constant of motion of system (29) which satisfies $d L\left(e_{M}\right)=0$, and

$$
\begin{aligned}
\left.d^{2} L\left(e_{M}\right)\right|_{W \times W}= & 2 \frac{M^{4}-k}{M^{4}-2 k} d y^{2} \\
& +2 \frac{\left(M^{4}-2 k\right)^{3}-16 k a^{2} M^{6}}{\left(M^{4}-2 k\right)^{3}} d z^{2} \\
& >0
\end{aligned}
$$

which finishes the proof.

Taking into account the results reported in [17-19, 3032], the Hamilton-Poisson realization of system (29) allows its study from the nonstandard Poisson geometry point of view. For this purpose, we consider the energy-Casimir mapping $\mathscr{E} \mathscr{C}: \mathbb{R}^{3} \rightarrow \mathbb{R}^{2}$ associated with the considered system; namely,

$$
\begin{aligned}
\mathscr{E} \mathscr{C} & (x, y, z)=(H(x, y, z), C(x, y, z)) \\
= & \left(\frac{x^{2}}{2}+\frac{k}{x^{2}}-\frac{y^{2}}{2}+2 a z, \frac{x^{2}}{2}+\frac{y^{2}}{2}+z^{2}\right) .
\end{aligned}
$$

Because the functions $H$ and $C$ given by (30) are constants of motion, the phase curves of system (29) are intersection of surfaces $H(x, y, z)=h$ and $C(x, y, z)=c$, where the pair $(h, c)$ belongs to the image of the energy-Casimir mapping given by

$$
\begin{aligned}
\operatorname{Im}(\mathscr{E} \mathscr{C}) & =\left\{(h, c) \in \mathbb{R}^{2} \mid(\exists)(x, y, z)\right. \\
& \left.\in \mathbb{R}^{3}: H(x, y, z)=h, C(x, y, z)=c\right\} .
\end{aligned}
$$

Moreover, the fiber of the energy-Casimir mapping $\mathscr{E} \mathscr{C}$ corresponding to an element $(h, c) \in \operatorname{Im}(\mathscr{E} \mathscr{C})$ is the set

$$
\begin{aligned}
& \mathscr{F}_{(h, c)} \\
& =\left\{(x, y, z) \in \mathbb{R}^{3} \mid H(x, y, z)=h, C(x, y, z)=c\right\},
\end{aligned}
$$

that is, the above-mentioned phase curve. 


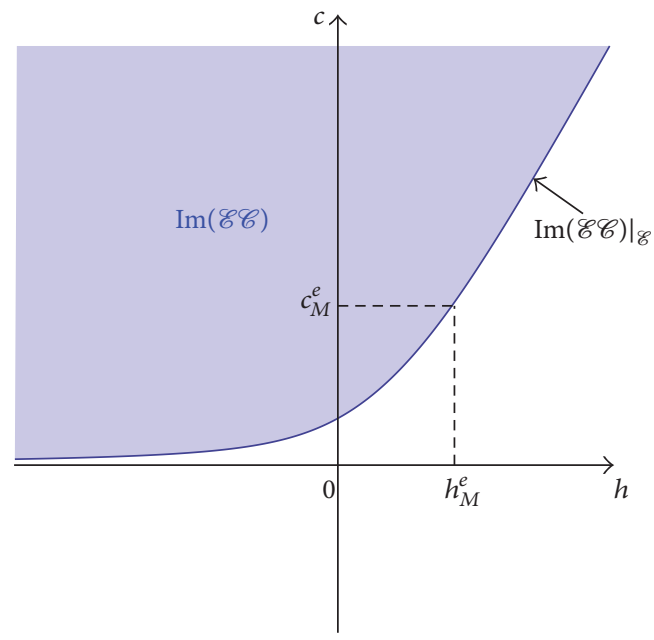

FIGURE 1: The image of the energy-Casimir mapping.

In the papers mentioned above, some results regarding the connections between the dynamics of some particular Hamilton-Poisson systems and the associated energyCasimir mappings were reported. More precisely, the boundary of the set $\operatorname{Im}(\mathscr{E} \mathscr{C}) \subsetneq \mathbb{R}^{2}$ is the union of the images of the stable equilibrium points through $\mathscr{E} \mathscr{C}$, and the image of the energy-Casimir mapping is convexly generated by these images. Moreover, for the points that belong to the interior of the set $\operatorname{Im}(\mathscr{E} \mathscr{C})$, the corresponding fibers are periodic orbits. We mention that in our case these connections hold.

In the next results, we present some properties of the image of the energy-Casimir mapping.

Proposition 7. Let $\mathscr{E} \mathscr{C}$ be the energy-Casimir mapping (44) associated with system (29). Then the boundary of the image of $\mathscr{E} \mathscr{C}$ is the union of the images of the stable equilibrium points through $\mathscr{E} \mathscr{C}$ (Figure 1):

$$
\begin{gathered}
\partial \operatorname{Im}(\mathscr{E} \mathscr{C})=\left\{\left(\frac{M^{2}}{2}+\frac{k}{M^{2}}+\frac{2 a^{2} M^{4}}{M^{4}-2 k}, \frac{M^{2}}{2}\right.\right. \\
\left.\left.+\frac{a^{2} M^{8}}{\left(M^{4}-2 k\right)^{2}}\right): M>0\right\} .
\end{gathered}
$$

Proof. Let $M>0$ and let $e_{M}:=\left(M, 0, a M^{4} /\left(M^{4}-2 k\right)\right) \epsilon$ $\mathscr{E}, a \in \mathbb{R}, k \in(-\infty, 0)$. Then $\mathscr{E} \mathscr{C}\left(e_{M}\right)=\left(h_{M}^{e}, c_{M}^{e}\right)$, where $h_{M}^{e}=M^{2} / 2+k / M^{2}+2 a^{2} M^{4} /\left(M^{4}-2 k\right)$ and $c_{M}^{e}=M^{2} / 2+$ $a^{2} M^{8} /\left(M^{4}-2 k\right)^{2}$. It is obvious that $\left(h_{M}^{e}, c_{M}^{e}\right) \in \operatorname{Im}(\mathscr{E} \mathscr{C})$ for every $M>0$. We prove that $\left(h, c_{M}^{e}\right) \notin \operatorname{Im}(\mathscr{E} \mathscr{C})$ for every $h>$ $h_{M}^{e}$. By definition (45), we have

$$
\begin{aligned}
\frac{x^{2}}{2}+\frac{k}{x^{2}}-\frac{y^{2}}{2}+2 a z= & \frac{M^{2}}{2}+\frac{k}{M^{2}}+\frac{2 a^{2} M^{4}}{M^{4}-2 k}+h \\
& -h_{M}^{e}, \\
\frac{x^{2}}{2}+\frac{y^{2}}{2}+z^{2} & =\frac{M^{2}}{2}+\frac{a^{2} M^{8}}{\left(M^{4}-2 k\right)^{2}} .
\end{aligned}
$$

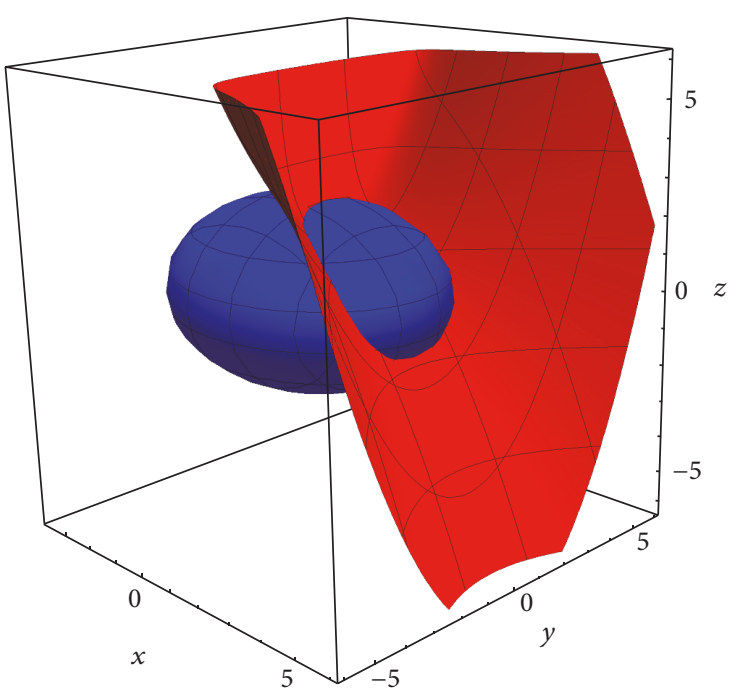

FIgURE 2: Periodic orbit $\left(k=-1, a=1, M=2, h=h_{M}^{e}\right.$, and $\left.c=c_{M}^{e}+4\right)$.

Multiplying (48) by $-M^{4} /\left(M^{4}-2 k\right)$ and then adding the result to (49), we obtain, after straightforward computations,

$$
L(x, y, z)=\left(h_{M}^{e}-h\right) \frac{M^{4}}{M^{4}-2 k},
$$

where $L$ is given by (38). Taking into account the properties of the function $L$, we obtain that there is no $(x, y, z)$ such that $\mathscr{E} \mathscr{C}(x, y, z)=\left(h, c_{M}^{e}\right)$ for every $h>h_{M}^{e}$; that is, $\left(h, c_{M}^{e}\right) \notin$ $\operatorname{Im}(\mathscr{E} \mathscr{C})$ for every $h>h_{M}^{e}$. Analogously we obtain that $\left(h_{M}^{e}, c\right) \notin \operatorname{Im}(\mathscr{E} \mathscr{C})$ for every $c<c_{M}^{e}$. It is clear that there is $y$ such that $\mathscr{E} \mathscr{C}\left(M, y, a M^{4} /\left(M^{4}-2 k\right)\right)=(h, c)$ in the case where $h<h_{M}^{e}$ and $c>c_{M}^{e}$. Moreover, $\mathscr{E} \mathscr{C}(x, y, z)=\left(h_{M}^{e}, c_{M}^{e}\right)$ if and only if $(x, y, z)=e_{M}$. In conclusion, the assertion has been proven.

Remark 8. By the proof of Proposition 7, we deduce that the image of the energy-Casimir mapping is convexly generated by the images of the stable equilibrium points through $\mathscr{E} \mathscr{C}$ (Figure 1). Furthermore, if $(h, c) \in \partial \operatorname{Im}(\mathscr{E} \mathscr{C})$, then the corresponding fiber is a set with exactly one element, namely, a stable equilibrium point.

Remark 9. The implicit equations of the corresponding fiber of a pair $(h, c)$ that belongs to the interior of the set $\operatorname{Im}(\mathscr{E} \mathscr{C})$ are

$$
\begin{aligned}
\frac{x^{2}}{2}+\frac{k}{x^{2}}-\frac{y^{2}}{2}+2 a z & =h, \\
\frac{x^{2}}{2}+\frac{y^{2}}{2}+z^{2} & =c .
\end{aligned}
$$

Such an intersection is presented in Figure 2. Taking into account Proposition 6, we conclude that this fiber is a periodic orbit. 


\section{Conclusions}

In this paper, integrable deformations of an integrable version of the Rikitake system were constructed, and HamiltonPoisson realizations of these new systems of differentiable equations were presented. Because it is an open problem to give the connections between the dynamics of such systems and the corresponding energy-Casimir mappings, the choice of certain deformation functions furnishes new examples that confirm or maybe infirm the properties reported for some particular systems. In this work, the deformation functions were chosen such that the Rikitake system is nonlinearly stabilized about its equilibrium points. In this case, the expected properties of the energy-Casimir mapping hold.

As future studies, the control and synchronization between new particular integrable deformations of the Rikitake system and original Rikitake system can be considered.

\section{Conflicts of Interest}

The author declares that there are no conflicts of interest regarding the publication of this paper.

\section{References}

[1] T. Rikitake, "Oscillations of a system of disk dynamos," Mathematical Proceedings of the Cambridge Philosophical Society, vol. 54, no. 1, pp. 89-105, 1958.

[2] H. K. Moffatt, "A self-consistent treatment of simple dynamo systems," Geophysical \& Astrophysical Fluid Dynamics, vol. 14, no. 1, pp. 147-166, 1979.

[3] Y. Feng, Z. Wei, U. E. Kocamaz, A. Akgül, and I. Moroz, "Synchronization and electronic circuit application of hidden hyperchaos in a four-dimensional self-exciting homopolar disc dynamo without equilibria," Complexity, vol. 2017, Article ID 7101927, 11 pages, 2017.

[4] Z. Wei, I. Moroz, J. C. Sprott, A. Akgul, and W. Zhang, "Hidden hyperchaos and electronic circuit application in a $5 \mathrm{D}$ selfexciting homopolar disc dynamo," Chaos: An Interdisciplinary Journal of Nonlinear Science, vol. 27, no. 3, Article ID 033101, 13 pages, 2017.

[5] K. Ito, "Chaos in the Rikitake two-disc dynamo system," Earth and Planetary Science Letters, vol. 51, no. 2, pp. 451-456, 1980.

[6] J. Llibre and X. Zhang, "Invariant algebraic surfaces of the Rikitake system," Journal of Physics. A. Mathematical and General, vol. 33, no. 42, pp. 7613-7635, 2000.

[7] C. Valls, "Rikitake system: analytic and Darbouxian integrals," Proceedings of the Royal Society of Edinburgh. Section A. Mathematics, vol. 135, no. 6, pp. 1309-1326, 2005.

[8] C. Lăzureanu and T. Bînzar, "On the symmetries of a Rikitake type system," Comptes Rendus Mathematique, vol. 350, no. 9-10, pp. 529-533, 2012.

[9] T. McMillen, "The shape and dynamics of the Rikitake attractor," The Nonlinear Journal, vol. 1, p. 10, 1999.

[10] J. Llibre and M. Messias, "Global dynamics of the Rikitake system,” Physica D. Nonlinear Phenomena, vol. 238, no. 3, pp. 241-252, 2009.

[11] U. E. Vincent, "Synchronization of Rikitake chaotic attractor using active control," Physics Letters A, vol. 343, no. 1-3, pp. 133$138,2005$.
[12] I. Pehlivan and Y. Uyaroğlu, "Rikitake attractor and it's synchronization application for secure communication systems," Journal of Applied Sciences, vol. 7, no. 2, pp. 232-236, 2007.

[13] X. Jian, "Anti-synchronization of uncertain Rikitake systems via active sliding mode control," International Journal of Physical Sciences, vol. 6, no. 10, pp. 2478-2482, 2011.

[14] V. Vembarasan and P. Balasubramaniam, "Chaotic synchronization of Rikitake system based on T-S fuzzy control techniques," Nonlinear Dynamics. An International Journal of Nonlinear Dynamics and Chaos in Engineering Systems, vol. 74, no. 1-2, pp. 31-44, 2013.

[15] S. Vaidyanathan, C. K. Volos, and V.-T. Pham, "Analysis, control, synchronization and spice implementation of a novel 4-d hyperchaotic rikitake dynamo system without equilibrium," Journal of Engineering Science and Technology Review, vol. 8, no. 2, pp. 232-244, 2015.

[16] S. Vaidyanathan, V.-T. Pham, and C. K. Volos, "A 5-D hyperchaotic Rikitake dynamo system with hidden attractors," European Physical Journal: Special Topics, vol. 224, no. 8, Article ID A1575, pp. 1575-1592, 2015.

[17] R. M. Tudoran, A. Aron, and S. Nicoara, "On a Hamiltonian version of the Rikitake system," SIAM Journal on Applied Dynamical Systems, vol. 8, no. 1, pp. 454-479, 2009.

[18] C. Lăzureanu and T. Bînzar, "A Rikitake type system with quadratic control," International Journal of Bifurcation and Chaos in Applied Sciences and Engineering, vol. 22, no. 11, Article ID 1250274, 14 pages, 2012.

[19] T. Bînzar and C. Lăzureanu, "A Rikitake type system with one control," Discrete and Continuous Dynamical Systems, Series B, vol. 18, no. 7, pp. 1755-1776, 2013.

[20] A. Galajinsky, "Remark on integrable deformations of the Euler top," Journal of Mathematical Analysis and Applications, vol. 416, no. 2, pp. 995-997, 2014.

[21] C. Lăzureanu, "On the Hamilton-Poisson realizations of the integrable deformations of the Maxwell-Bloch equations," Comptes Rendus Mathematique, vol. 355, no. 5, pp. 596-600, 2017.

[22] A. Ballesteros, A. Blasco, and F. Musso, "Integrable deformations of Lotka-Volterra systems," Physics Letters. A, vol. 375, no. 38, pp. 3370-3374, 2011.

[23] A. Ballesteros, A. Blasco, and F. Musso, "Integrable deformations of Rossler and Lorenz systems from Poisson-Lie groups," Journal of Differential Equations, vol. 260, no. 11, pp. 8207-8228, 2016.

[24] G. Chen and X. Dong, "From chaos to order-perspectives and methodologies in controlling chaotic nonlinear dynamical systems," International Journal of Bifurcation and Chaos, vol. 3, no. 6, pp. 1363-1409, 1993.

[25] A. E. Cook and P. H. Roberts, "The Rikitake two-disc dynamo system," Mathematical Proceedings of the Cambridge Philosophical Society, vol. 68, no. 2, pp. 547-569, 1970.

[26] Y. Hardy and W.-H. Steeb, "The Rikitake two-disk dynamo system and domains with periodic orbits," International Journal of Theoretical Physics, vol. 38, no. 9, pp. 2413-2417, 1999.

[27] A. V. Bolsinov and A. V. Borisov, "Compatible Poisson brackets on Lie algebras," Mathematical Notes, vol. 72, no. 1, pp. 10-30, 2002.

[28] A. M. Lyapunov, Problème Général de la Stabilité du Mouvement, vol. 17, Princeton University Press, Princeton, NJ, USA, 1949.

[29] P. Birtea, M. Puta, and R. M. Tudoran, "Periodic orbits in the case of a zero eigenvalue," Comptes Rendus Mathematique, vol. 344, no. 12, pp. 779-784, 2007. 
[30] C. Lăzureanu and T. Bînzar, "Some geometrical properties of the Maxwell-Bloch equations with a linear control," in Proceedings of the in Proceedings of the 13th International Conference of Mathematics and Its Applications, pp. 151-158, Timisoara, Romania, November 2012.

[31] T. Bînzar and C. Lăzureanu, "On some dynamical and geometrical properties of the Maxwell-Bloch equations with a quadratic control," Journal of Geometry and Physics, vol. 70, pp. 1-8, 2013.

[32] C. Lăzureanu and T. Bînzar, "Symmetries and properties of the energy-Casimir mapping in the ball-plate problem," Advances in Mathematical Physics, vol. 2017, Article ID 5164602, 13 pages, 2017. 


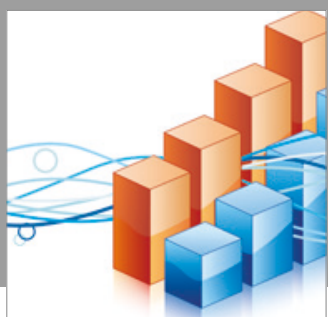

Advances in

Operations Research

vatersals

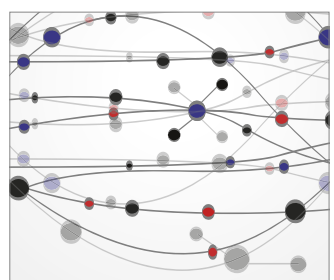

\section{The Scientific} World Journal
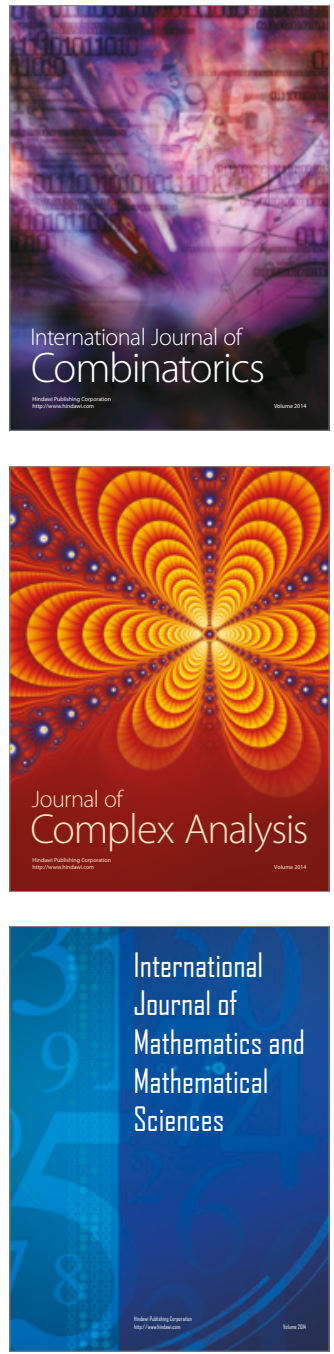
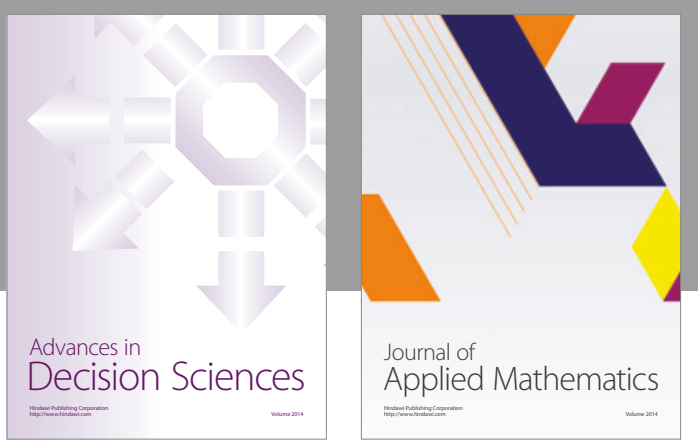

Algebra

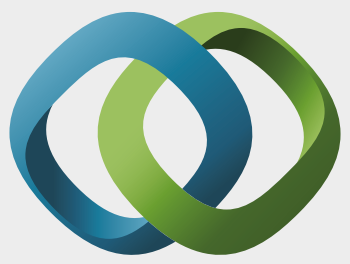

\section{Hindawi}

Submit your manuscripts at

https://www.hindawi.com
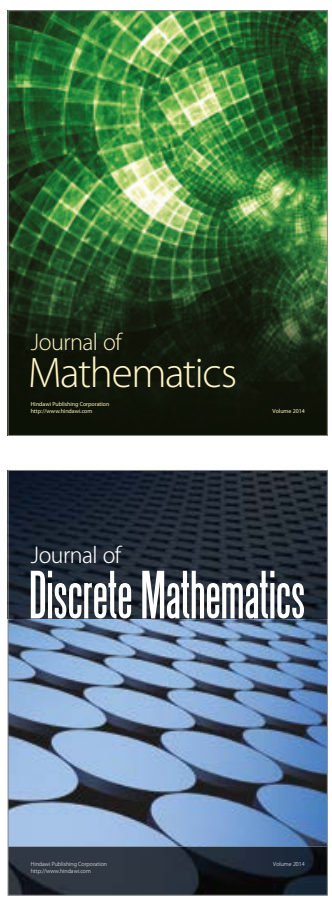

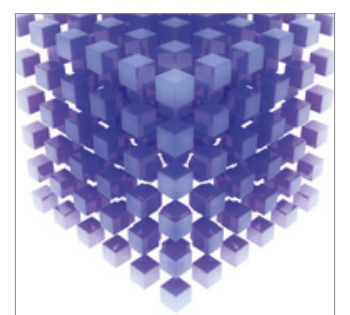

Mathematical Problems in Engineering
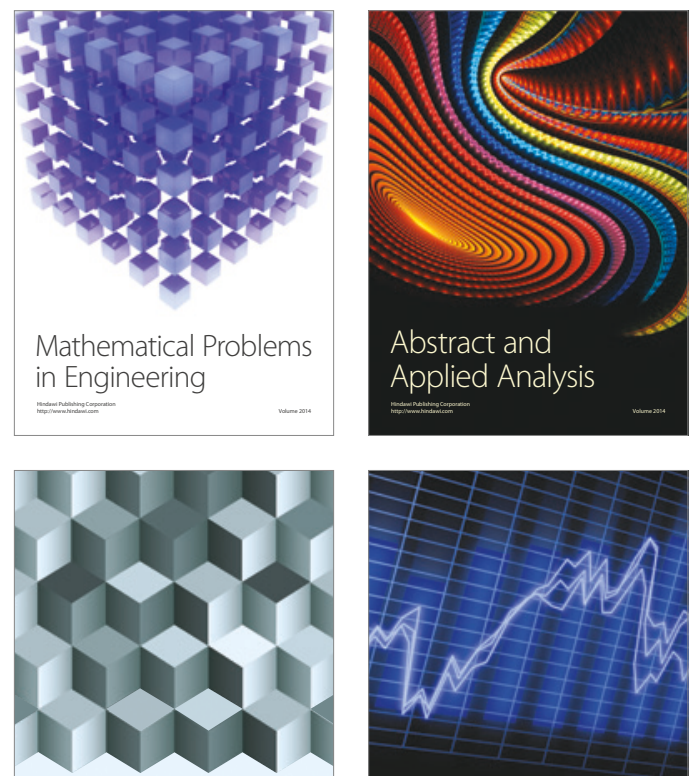

Journal of

Function Spaces

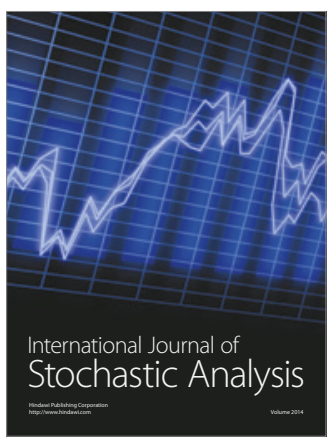

Probability and Statistics
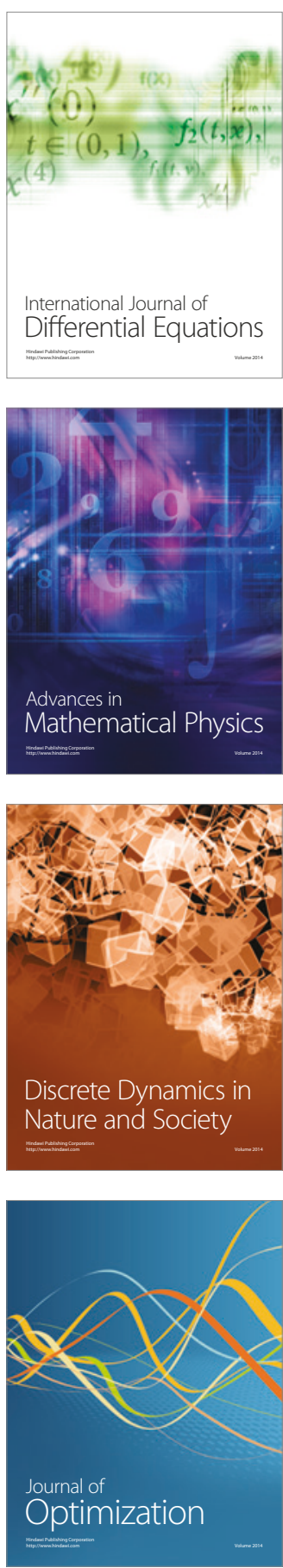